Recibido: 1 mayo 2019 | Aceptado: 25 octubre 2019 | Publicado: 1 diciembre 2019

Cita: Fuentes, C. (2019). En lo que cabe y la limitación de la aserción. Normas, 9, 101-113. doi:

https://doi.org/10.7203/Normas.v9i1.16165

\title{
EN LO QUE CABE Y LA LIMITACIÓN DE LA ASERCIÓN
}

EN LO CABE AND THE LIMITS OF ASSERTION

\section{Catalina Fuentes Rodríguez}

Universidad de Sevilla

Resumen

El presente trabajo analiza la expresión en lo que cabe, construcción en proceso de fijación como operador discursivo que muestra cierta complejidad en su construcción y comportamiento macrosintácticos. Cumple algunos requisitos de los llamados operadores discursivos, pero coexiste con la construcción libre. Su valor es el de limitar una valoración y reducir la fuerza argumentativa de su alcance. Este contenido procedimental se desarrolla a partir del contenido semántico originario y de las funciones que cumple en el discurso.

PALABRAS CLAVE: Operador discursivo, construcción, limitación de la aserción, valoración, macrosintaxis
Abstract

The present work analyzes the expression en lo que cabe, construction in process of fixation as discursive operator that shows certain complexity in its construction and macrosyntactic behavior. It satisfies some requirements of the so-called discursive operators, but coexists with free construction. Its value is to limit an assessment and reduce the argumentative force of its scope. This procedural meaning evolves from the original semantic content and the functions it fulfils in the discours

KEY WORDS: Discourse marker, construction, limit of asertion, evaluation, macrosyntax 


\section{INTRODUCCIÓN}

El trabajo que presento para este número monográfico, en homenaje a A.Briz, se detiene en una forma que está en proceso de fijación como operador discursivo y muestra cierta complejidad en su construcción y comportamiento macrosintáctico.Se inscribe en un proyecto más amplio ${ }^{1}$, que reflexiona sobre el proceso creativo de la lengua en este campo de las partículas discursivas, que tanto ha ocupado al grupo Val.Es.Co. y a su director (Briz-Pons-Portolés 2008-actualidad). Al mismo tiempo, nos obliga a reconsiderar los conceptos limitados que la sintaxis hasta ahora ha venido manejando. Muchos de ellos resultan a veces totalmente ineficaces para albergar la multidimensionalidad del discurso.

La metodología empleada es la Lingüística pragmática (Fuentes Rodríguez 2017ª (2000)), en la que venimos trabajando varios años. Concretamente partimos de un enfoque macrosintáctico (Berrendonner 1990, 2002,2003, Blanche Benveniste 2002,2003, Avanzi 2017) ya que entendemos que desde un enfoque global podemos acercarnos a la descripción de formas y expresiones que van apareciendo en el uso comunicativo y que se mueven en un plano supraoracional (Deulofeu 2016, Fuentes Rodríguez 2017b). Estas formas suelen referirse, muchas veces, a las coordenadas "thetical" (Kaltenböck-HeineKuteva 2011) y están próximas a los llamados modificadores en la Gramática-DiscursivoFuncional (Hengeveld-Mackenzie 2008) y a las "partículas discursivas" del grupo Val.Es.Co. Para Kaltenböck-Heine-Kuteva (2011) exigen una nueva gramática, separada de la tradicional "sentence gramar". Por nuestra parte, consideramos que hay que extender la gramática e integrar el discurso, en una tarea que no admite más demoras.

Nos centraremos en la forma en lo que cabe, estructura muy cercana a otras que limitan la aserción (vid. Fuentes Rodríguez 2019, e.p.) y reducen la fuerza argumentativa de su alcance. Utilizaremos las bases de datos de la RAE, CREA, CORPES y CORDE, en este primer momento de la investigación.

\section{OPERADORES DISCURSIVOS}

En la metodología que estamos siguiendo en el proyecto partimos de la distinción entre conectores y operadores discursivos, establecida ya en Fuentes Rodríguez (2003). Estos últimos son partículas o expresiones que no establecen una conexión entre dos miembros como los conectores, sino que se mueven en el ámbito del enunciado, o incluso pueden constituir enunciado independiente (como los modales en intervenciones reactivas, cfr. Quirk et al. 1972, Greenbaum 1969). Estos segmentos no afectan a la predicación oracional, no tienen función dentro de ella, aunque su alcance puede ser una oración o un segmento. El contenido que aportan y la función discursiva a la que apuntan se mueven en el plano procedimental. Realizan una indicación relativa a la enunciación, modalidad (relacionados con el hablante), argumentación u organización informativa del texto (relativos al oyente) (Fuentes Rodríguez 2018a). En muchas ocasiones se manifiesta de manera muy clara su multidimensionalidad: afecta a diversos planos de manera simultánea unas veces,

\footnotetext{
${ }^{1}$ Este trabajo forma parte del proyecto FFI 2017-82898P, financiado por el Mineco y fondos FEDER.
} 


$$
\text { En lo que cabe y la limitación de la aserción | C. Fuentes }
$$

otras de manera jerarquizada. Un contenido aparece como principal y otro con información de segundo plano.

En cuanto a la distribución sintáctica, el elemento o expresión tiene movilidad en la frase y puede aparecer en posición inicial, final o intermedia, ligado o no a su ámbito desde el punto de vista entonativo y en distribución antepuesta o pospuesta a este.Veamos unos casos:

(1a) Hasta en verano lleva chaqueta

Hasta aparece en posición antepuesta al ámbito, sin separación entonativa, posición inicial

(1b) Lleva la chaqueta en verano solo/solo en verano

Solo puede preceder o seguir a su alcance, sin separación entonativa en este caso e intercalado

(1c) Lleva una chaqueta muy bonita, francamente

Francamente está pospuesto, en posición final, con separación entonativa

(1d) Francamente, llevas una chaqueta muy bonita

Francamente aparece al inicio del enunciado, formando un grupo entonativo independiente.

El ámbito en (1c) y (1d) es todo el contenido del enunciado. En (1a) y (1b) es un segmento oracional.

El objetivo del proyecto, y de este trabajo, es conocer el camino sintáctico sufrido (o que están sufriendo) construcciones que están en vías de fijación como nuevos operadores discursivos. No solo detectamos los nuevos elementos sino, sobre todo, describimos la dinámica macrosintáctica, lo que nos permite elaborar conclusiones sobre las relaciones que se establecen en el discurso, la interacción entre función, posición, entonación, limitación de ámbito, extraproposicionalidad, que nos permita entender mejor la gramática/pragmática de estas unidades y, sobre todo, nos permita conocer la sintaxis del discurso. En el punto siguiente veremos de la mano de en lo que cabe los pasos del proceso de fijación que consideramos.

\section{EN LO QUE CABE: DESCRIPCIÓN MACROSINTÁCTICA}

En lo que cabe es una construcción sintagmática, formada por una preposición y una estructura de relativo sustantivada. Incluye el verbo caber, cuyo contenido espacial sufre un proceso de cambio en esta y otras expresiones. Véase su uso en perífrasis como cabe considerar, cabe admitir..., ligadas generalmente al registro formal.

Es una construcción en proceso de fijación, que cumple algunos requisitos de los llamados operadores discursivos, pero coexiste con la construcción libre. Aparece a veces integrada en la oración y otras como elemento periférico sin ningún complemento verbal. En estos casos forma grupo entonativo independiente. Esta diversidad no es exclusiva de este 
segmento, pero al ser una construcción aún no completamente fijada, es más frecuente encontrar mayor diversidad sintagmática. A la vez pone sobre la mesa la discusión sobre la relevancia del concepto de aislamiento fónico-entonativo ligado a las partículas discursivas (Brinton 2010, Dehé 2009), ya que en el caso de los operadores, sobre todo, necesita una revisión. Que un elemento sea extraproposicional (Dik 1997) no implica que deba aparecer con aislamiento entonativo, aunque esta característica sea más discriminante que la integración.

En cuanto al contenido procedimental que esta construcción establece en su función como operador se mueve entre lo enunciativo y lo argumentativo y se acerca los complementos de ámbito o tópicos (Gutiérrez Ordóñez 1997).

En esta función se acerca a otra forma, dentro de lo que cabe, y a expresiones que apuntan a la modalidad: en lo posible, en la medida de lo posible, en la medida de mis /tus/sus posibilidades. ${ }^{2}$

Nuestra reflexión se dirige en dos sentidos: el proceso de fijación (gramaticalización, pragmaticalización, construccionalización ${ }^{3}$ ) que sigue, así como la discusión sobre su función macrosintáctica en el enunciado.

\subsection{Complemento de ámbito}

En lo que cabe + complemento es una expresión que actúa como complemento circunstancial de lugar si los complementos que lleva cabe son locativos ("en lo que cabe en una cesta"), pero pasa a utilizarse con valor nocional en el sentido de "en lo relativo a, en lo referente a...". Establece el ámbito de referencia, como encontramos en el siguiente ejemplo del Corpes. Actúa como circunstancial dependiente del verbo de la oración (enfocarse):

\footnotetext{
(2) (...) se emociona, porque habla de la recuperación de una simbología que en nuestra generación era muy importante y que fue degradada por la dictadura, pero que de a poco, con el acompañamiento de las fuerzas armadas en el proceso democrático, se va acomodando", reseña él. Y enfoca, ahora sí, en el presente. En lo que cabe contar hoy. (CORPES, Vitale, Cristian: "Yo sentía que no debía editar nunca estos poemas"». Página/12. Rosario: pagina12.com.ar, 2011-1124.)
}

La oración de relativo sustantivada puede llevar cualquier complemento. Es una construcción libre y el sintagma introducido por en lo que cabe tiene más recorrido y justifica los valores de la construcción. En el caso siguiente es complemento del verbo caracterizar:

(3) Creo que la idea de un "nomadismo estético", desarrollada por Mauricio Bravo Carreño en relación a obras que ocupan el viaje como "soporte de producción imaginal", permite caracterizar a Road Trip I especialmente en lo que cabe a su rol terapéutico. (CORPES, Benavente Morales, Carolina: «Road trip i stgo-baires-stgo 2009: emplazamiento y cuerpo nómade de la obra». Escáner cultural. Santiago de Chile: escaner.cl, 2009-07).

\footnotetext{
${ }^{2}$ Vid Fuentes Rodríguez (2019a y b)

${ }^{3}$ Traugott (1995), Company (2003), Dostie (2004), Diewald (2011),Claridge-Arnovick (2010)
} 
Este complemento establece el contexto en el que puede aplicarse una predicación ("en lo que se refiere, en lo que entra en su rol terapéutico, en cuanto a su rol terapéutico"). En este caso podríamos hablar ya de una construcción (Goldberg 1996) que se fija como introductor de un complemento: en lo que cabe a+ s.n. (preposición compleja). Es una estructura formal con una función y categoría nueva. A su vez se produce también un cambio de contenido. Es un complemento de ámbito de una aserción, un complemento periférico dentro de un enunciado.

\subsection{Complemento periférico de la enunciación}

El siguiente contexto en el que encontramos esta expresión es como complemento periférico (Fuentes Rodríguez 2012, 2014, 2018b): aparece como un complemento del decir (Ducrot 1984, Fuentes Rodríguez 2004, López Martín 2017). En este contexto se combina con el verbo hablar, decir, llamar.

\footnotetext{
(4)-En táctica militar, es cierto. Pero calculábamos que serían dos pelotones, máximo. Si no se rendían, incendiaríamos el cuartel para obligarlos a salir y tendríamos copadas las salidas. Sobre esa base el triunfo era fácil, en lo que cabe hablar de facilidad en estas cuestiones. (CORPES, Montemayor, Carlos: Las armas del alba. México D. F.: Joaquín Mortiz, 2003).
}

La construcción "en lo que cabe (+verbo de habla)" tiene una función en el margen derecho y desarrolla un contenido enunciativo. Al actuar como complemento de enunciación se liga al acto de emitir el enunciado, por lo que puede usarse con la forma en presente (cabe) a pesar de estar refiriéndose a un contexto de pasado. El sentido usado en este empleo es la acepción 4 que recoge el Diccionario de la Lengua Española: "en lo que es posible hablar". Es un primer paso a lo epistémico, a lo procedimental. De ahí su relación con la modalidad o la regulación de la aserción.

\subsection{Operador}

El empleo como operador aparece en los casos en que el segmento no lleva complementos, forma un grupo entonativo independiente, una unidad fraseológica, sin posibilidad de extensión, con un contenido único procedimental. Es el mayor grado de fijación y de liberación sintáctica y la menor dependencia del contexto.

\footnotetext{
(5)-Buenos días, Hans. ¿Cómo te han tratado?

-Bien, en lo que cabe. La celda es poco confortable. (CORPES, Trujillo Muñoz, Gabriel: Trenes perdidos en la niebla. México, D. F.: Jus, 2010).
}

En lo que cabe es una estructura prácticamente fijada ya, que establece una limitación en la aserción. Actúa como un marco o punto de referencia de una información, en distribución parentética. Generalmente aparece pospuesto a su alcance y no siempre entre pausas, puede estar integrado en su grupo entonativo.

Este nuevo valor es el que adopta en lo que cabe como grupo, con un contenido procedimental y una función discursiva clara. En los corpus analizados (CREA, Corpes) aparece con adjetivos o calificaciones. Su alcance suele ser una valoración, cuyo límite establece. De ahí que generalmente aparezca tras adjetivos o en estructuras atributivas: ser +adjetivo, resultar+adjetivo, o sintagmas nominales. 


\title{
En lo que cabe y la limitación de la aserción | C. Fuentes
}

\begin{abstract}
(6)Durante quince años llevé una vida más o menos normal, en lo que cabe. Pero un día de mi adolescencia empecé a crecer sin medida ni control y a ser percibido como un fenómeno, una especie de monstruo inexplicable, y esta circunstancia coincidió con mi necesidad de hallar una forma de ganarme la vida. (CORPES, Jaramillo Levi, Enrique: «Secreto a voces». Secreto a voces. San José: Uruk, 2008)
\end{abstract}

En (6) aparece tras normal, y en (5) con adverbios como bien. Incluso puede ser frecuente en respuestas: ¿estás bien? sí, en lo que cabe, como en el ejemplo siguiente.

(7)-¿Pero le va bien?

-Pues en lo que cabe.(CORPES, Rodríguez Rodríguez Ángel : Cuando los macuelizos florecen. San

Pedro Sula: Pacura Editorial, 2010).

En (7) aparece sin la valoración. En el turno anterior se pregunta sobre un estado o proceso (ir bien) que se presupone en la respuesta: "pues me va bien en lo que cabe".

En (5)se presupone la predicación anterior y se limita: "me han tratado bien". Esta valoración se presenta circunscrita a lo que es posible, habitual o esperable. No se establece ningún marco o punto de referencia (en lo que cabe a ...), por lo que el hablante debe suponerlo. Es fruto del proceso de abstracción necesario en la gramaticalización (Company 2004). De este modo la expresión en lo que cabe adquiere ese contenido de "en lo posible en las circunstancias actuales". El punto de referencia es el contexto del habla.

Este nuevo contenido procedimental desarrollado va unido a la fijación como grupo, con independencia sintáctica y referencial de lo expresado en la oración.

En lo que cabe ya no es un complemento del verbo, no lleva complementos. Actúa como un grupo independiente, no establece dependencia con el verbo de la oración, consigue la extraproposicionalidad. Semánticamente no necesita punto de referencia en el que establecer su indicación de ámbito. Ahora alude a las coordenadas cognitivas generales, a las creencias del grupo. En este caso en lo que cabe reduce la valoración positiva de una acción a un nivel "relativo", más bajo en la escala.

Actúa, por su propia distribución, como un operador argumentativo con valor de reducción de la fuerza argumentativa. Esta función se entiende como una extensión desde su origen como sintagma enmarcador. Sus contextos sintagmáticos predominantes suelen ser, como hemos dicho, el pospuesto al alcance, aunque sea posible también la anteposición.

\section{(8)(...) y los jóvenes de los cafés literarios y la academia que se convertirían, en lo que cabe, en mandarines de la cultura, respetados por un establishment -compuesto, entre otros, por los antiguos enarcas- que les honraría, leería y comentaría, saboreando sus críticas radicales y sus manifiestos surrealistas, existencialistas o estructuralistas (CORPES, Pérez-Díaz, Víctor: Sueño y razón de América Latina. Política, cultura y sociedad civil en la gran transición. Madrid: Taurus, 2005).}

En este caso recae su acción sobre un sustantivo: "(convertirse en) mandarines de la cultura". La palabra mandarín significa aquí "iron. Persona influyente en los ambientes políticos, artísticos, literarios, sociales, etc. U. m. en pl.” (acepción 5 del DEL).

Es más frecuente en el interior del enunciado, antepuesto o pospuesto al elemento que constituye su alcance. Podríamos decir, pues, que ha pasado de la función marco del 


\section{En lo que cabe y la limitación de la aserción | C. Fuentes}

enunciado a un contenido procedimental limitador de una predicación y que, por tanto, tiene la versatilidad de aplicarse a cualquier elemento del mismo.

Al anteponerse puede intercalarse rompiendo la linealidad sintáctica, como en (9) en que recae sobre el sustantivo atributo: ser una anfitriona modelo.

(9) Ahora sí ya de refilón se añade que doña Zulema fue, en lo que cabe, una anfitriona modelo, a tal grado que se cansó de serlo, tal fue la resulta de recibir (sin cesar) parientes de aquél a lo largo de unos cinco años. (CORPES, Sada, Daniel: Casi nunca. Barcelona: Anagrama, 2008).

En esta posición intercalada focaliza el atributo. Puede incluso intercalarse entre sustantivo y adjetivo, como en el caso siguiente:

(10) Detrás de la barra, un hombre de cara desencajada pero modales, en lo que cabe, correctos, me hizo una seña de ¿Qué va a ser? Yo le hice otra de Lo estoy pensando. (CORPES, Ehrenhaus, Andrés: La seriedad. Barcelona: Mondadori, 2001).

Su función es restrictiva: limita el campo de aplicación de una valoración: Ios términos son siempre positivos, con lo que el movimiento dentro de la escala es una minoración, en dirección descendente, que rebaja la fuerza de lo dicho.

(11) laggo: Una historia ligera en lo que cabe. (CORPES, Chías, Édgar: Una merienda de negros:

8: No levantarás falsos testimonios). México D. F.: Pasodegato, 2009).

12)En mi buhardilla soy feliz en lo que cabe, si tú me faltas. Me gustan sus muebles pueblerinos,

los pañitos de punto de ganchillo que la señora a la que se la alquilo, pone sobre ellos, su tragaluz.

(CREA, Díaz, María Paz: Amor en Florencia. Brenes: Muñoz Moya Editores, 2002.)

Feliz pasa de ser un término situado en la zona positiva de la escala a rebajarse unos puntos y quedarse en la zona central o incluso en la negativa. Las inferencias provocadas son justo las contrarias: "no estoy feliz".

(13) Por otra parte, se considera una mujer feliz en lo que cabe "porque estoy segura".(CORPES,

Abarca, Lilia: «Silvia Pinal ama comer mariscos». El Universal.mx. México D. F.: eluniversal.com.mx,

2004-01-16)

El proceso de inferencia que provoca en lo que cabe puede ilustrarse con la figura que sigue. En lo que cabe establece una dirección escalar descendente ${ }^{4}$. El adjetivo está situado en una posición de la escala positiva, generalmente elevada, y con en lo que cabe, al relativizar la evaluación, se dirige hacia una zona más neutra o incluso baja.

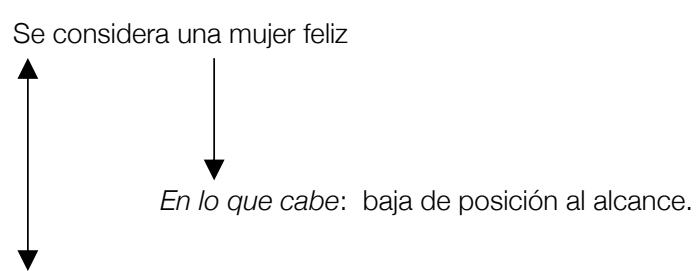

No encontramos adjetivos negativos en el corpus. De hecho, sería extraña una secuencia del tipo "Es malo en lo que cabe" o "Es un desastre en lo que cabe", frente a "Es muy

\footnotetext{
${ }^{4}$ Confróntese Fauconnier (1975) para el concepto de escala, Anscombre-Ducrot (1983) y Fuentes Rodríguez (2016) sobre las inferencias de ciertos operadores escalares.
} 
bueno en lo que cabe". "Es un hacha, en lo que cabe". "Es excelente, en lo que cabe". Tampoco admite términos que no sean evaluativos: "Es un ministro, en lo que cabe"*.

Podemos afirmar, pues, su función como un correctivo, limitador. Esta rebaja se produce al establecer un punto de referencia, no expreso, que cabe situar en lo considerado habitual, razonable en la situación de la que hablamos (soy feliz en lo que puede afirmarse en las circunstancias que tú conoces, es una historia ligera en lo que puede serlo en la situación considerada, es una anfitriona modelo en lo que puede esperarse). El operador, pues, obvia ese punto de referencia, los omite y sitúa en el marco cognitivo conocido por hablante y oyente.

Afirmamos, asimismo, su grado de fijación por su movilidad (antepuesta (10) o pospuesta (11)a su alcance). Puede aparecer también en parentéticos, como un comentario (Fuentes Rodríguez 2018). En el enunciado que sigue lo apoya un adverbio reafirmativo.

(14) Zapatero va a tener un par de oportunidades para departir de manera distendida -en lo que cabe, claro- con los informadores que habitualmente siguen los avatares políticos. (CORPES, Jáuregui, Fernando: "Zapatero, el de las grandes ojeras». Diario de Burgos. Burgos: diariodeburgos.es, 2008-12-15.)

Encontramos casos en que aparece como enunciado independiente en un turno interrumpido, modificando lo dicho, rebajando su fuerza:

(15) Yo no me acuerdo, pero bueno, claro, por supuesto, que si le piden la plata para tal cosa pero en realidad no sé, no yo no he tenido tampoco cosas así graves de decir no. No, la atención es buenaBueno, cuando yo En lo que cabe. Pues bueno, no sé. ¿Y quedó bien su marido después de la operación? Sí, gracias a Dios (CREA, Oral, CSMV, texto MDC2F)

En todos estos contextos en lo que cabe limita la aserción, actúa sobre una valoración que rebaja. El proceso semántico sufrido va desde: a) la indicación de marco locativo ("en lo que cabe en...") a marco nocional ("en lo que cabe en/a..."); b) ámbito del decir y c) reducción de la capacidad referencial del término que constituye su alcance. En este último estadio desarrolla un contenido procedimental basado en la inferencia provocada sobre la valoración escalar.

Sintácticamente los diferentes contextos de aparición revelan un paso progresivo de lo periférico al operador. Comienza con funciones de complemento de ámbito dependiente directamente del verbo de la oración, pasa por una función periférica como complemento de la enunciación y termina como grupo fijado, desarrollando una mayor libertad posicional y funcional. El operador argumentativo ya no depende del verbo de la oración, es un elemento macrosintáctico que puede afectar a cualquier segmento del enunciado. Este sería su alcance y sobre él, una valoración de cualquier tipo, puede actuar la expresión, antepuesta o pospuesta.

\section{El proceso sería:}

c. de ámbito-c. enunciación (periférico)--- operador de limitación (descenso escalar en la inferencia) 
El marco o punto de referencia es el conocimiento compartido. Su contenido pasa de : [valoración positiva+ en lo que cabe- marco] a una reducción y una reorientación: hacia lo negativo.

\title{
3.4 Fijación
}

Sobre su grado de fijación podemos afirmar que aparece bastante avanzado en su proceso, ya que forma una unidad sintáctica, que no permite inserción de ningún otro elemento. Tiene movilidad de posición con respecto a su alcance. Sin embargo, hemos hemos encontrado algunos casos de en lo que cabía, concretamente dos en todo el CREA, en interior de enunciado. El valor es semejante al de en lo que cabe, aunque ahora no se elige el presente, como forma ligada al momento de la enunciación, sino que hay una adaptación temporal a la narración. Esta consecutio temporum muestra un grado aún no completo en la fijación. En (16) se acerca a la función como operador y en (17) introduce un complemento de ámbito.

\begin{abstract}
(16)Con un bagaje bien definido de la historia, deambula por su obra con frecuencia obsesionante para dar una exacta cuenta, el lujo de detalles en los cuadros históricos de su país en el turbulento periodo de 1812 a 1831, presentando sus personajes "con todos sus pelos y señales", como vulgarmente se dice, "vestidos con el traje que llevaron en vida, la mayor parte bajo su nombre y apellidos verdaderos, hablando el mismo lenguaje que usaron en las escenas históricas en que figuraron, copiando en lo que cabía "d'après nature", su fisonomía física y moral, a fin de que aquellos que los conocieron de vista o por tradición los reconozcan sin dificultad y digan cuando menos "el parecido es innegable"". (CREA, Hernández de Norman, Isabel: La novela criolla en las Antillas, 1977)

(17) Y lo que se nos cuenta es su experiencia de joven rebelde -en lo que cabía en la Polonia de 1964-, que ha formado con unos amigos un grupo musical en lo que ellos creen ser la línea de sus ídolos, Los Beatles, que se enamora por primera vez y que por primera vez tomará conciencia -con perdón por la frase- de que las cosas a su alrededor no marchan como debieran, de que la libertad, en todas sus acepciones, es algo tan ansiado como lejano. (CREA, ABC, 16/06/1996)
\end{abstract}

En estos casos parece mantener el valor de en lo que era posible, más ligado al contenido originario de la estructura, no como construcción fijada.

\section{VALOR MODAL}

Hay otro contexto, sin embargo, en el que en lo que cabe no señala tan claramente esta limitación y rebaja en la inferencia, provocando una disminución, sino que su empleo se mueve en el ámbito de la posibilidad, como combinatoria libre donde cabe se usa con este contenido léxico. Encontramos algún caso con adjetivos como impermeable, en un contexto prospectivo.

(18) Lo que hay que exigir al calzado de montaña es: que sea fuerte, que sea cómodo, que sea impermeable (en lo que cabe), que sea cálido (en tiempo frío).(CREA, A.Faus:Andar por las montañas, 1999)

En lo que cabe puede ser interpretado como "si es posible," o "en la medida de lo posible". No parece que indique limitación escalar. El otro contexto en el que lo hemos encontrado es con el verbo evitar. La interpretación escalar sería: "evita poco" o "no lo evita". No es un contexto valorativo positivo como en el resto de empleos considerados en 3. La interpretación modal, derivable del contenido de caber, sería "en lo posible" o "si es posible". Esta construcción aparece sin complementos en distribución semejante a la de 
un operador. El efecto sigue siendo reducir la fuerza asertiva, incluso ponerla en duda o hacerla depender de las posibilidades de realización. El contenido de orientación negativa se piede.

(19) Modernamente, algunos crampones están recubiertos de una pintura que evita -en lo que cabela formación de zuecos. Más útiles son unas piezas intermedias anti-zuecos como plantillas, que evitan este peligro. (CREA,A.Faus:Andarse por las montañas, 1999)

Esta deriva hacia el contenido modal está implícita en el uso del núcleo léxico de la construcción, caber. El marco puede acercarse a la posibilidad y al efecto atenuador. Esta relación será tratada en otro trabajo (vid Fuentes Rodríguez 2019b).

\title{
5 RESULTADOS EN LOS CORPUS
}

Las primeras apariciones de en lo que cabe en Corde se remontan a 1548:

\begin{abstract}
(20) Proveyóse esta conquista para sacar gente de esta tierra de la que ha servido á S. M. en esta jornada, la cual ya empieza á ir entendiendo que no se les puede dar otro remedio, é con lo que el tiempo puede é con haberme esforzado á mostrarles alguna esquiveza para que no con tanta familiaridad me importunen sobre lo que no puedo ni tengo que dalles, aunque de tal manera es esto que en lo que cabe no les dejo de mostrar el amor grande que les tengo, como á personas que en esta jornada me han hecho buena compañía é me han amado; van ya mejorando en conocer el respeto que á los ministros de S. M. é temor á su justicia deben tener, é toman cuidado de buscar su propio remedio. (CORDE, Carta del licenciado Pedro de la Gasca, 1548).
\end{abstract}

Entre ellas encontramos el uso de esta expresión con valor limitador, pero también otras en las que sigue siendo un sintagma enmarcador: "en lo que cabe en indias", "en lo que cabe al principal cargado". En el s. XVIII de 8 apariciones de la expresión en lo que cabe, 4 son del operador.

(21) Está adelantada, en lo que cabe, la gente (CORDE, R.de la Cruz: El petrimetre, 1764)

(22) Los trabajadores lo hacen con el lodo a media pierna, ellos sufren esta incomodidad pero aprovecha su trabajo, y lo llevan con gusto en lo que cabe.

Por lo mismo, no puede nuestro intendente venir y será bueno lo haga estándolo el tiempo. (CORDE,

D.de Vargas y Carvajal: Carta a Rodríguez Campomanes, 1772)

Junto a ellos, hay un caso en 1761 en el que aparece coordinada con otra construcción, lo que revela su comportamiento como complemento circunstancial:

(23) En cuanto al mérito de la obra, te puedo decir que es de las más útiles que han salido en España en este siglo, y el autor ha desempeñado, en lo que cabe y puede hacer un hombre solo en este género de estudios, la vasta idea que se propuso. Hay en esta obra algunas disertaciones que están muy bien escritas.(CORDE, M.Lanz de Casafonda: Diálogos de Chindulz, 1761)

En los siglos XIX y XX encontramos otros 38 casos. De ellos hay 6 en que actúa como construcción libre, complemento del verbo de la oración y con otra extensión sintáctica ("en lo que cabe dentro de las cosas humanas", "en lo que cabe hacerlo", y "en lo que cabe por su edad") y tres de en lo que cabe llamar, ligado a la enunciación.

Es un elemento, pues, que empieza a desarrollarse en el s. XVIII, aunque tiene una aparición en el XVI, y que ya toma más impulso en el XIX y XX. De los 38 casos, 7 son del XIX y el resto del $X X$. De todos esos casos solo 13 son del operador. 
En CREA encontramos 8 casos, de ellos 7 del marcador.Y en Corpes 11 de 14. Los otros 3 son del complemento de ámbito.

\begin{tabular}{|l|l|l|}
\hline CORDE: 13 (de 38) & CREA & CORPES \\
\hline S-XVI:1 & 7 (de 8) & 11 (de 14). \\
s.XVIII: 4 & & \\
S.XIX-XX: 32 (de 38) & & \\
\hline
\end{tabular}

Es, pues, una expresión que está en los primeros pasos de la fijación, que no está muy extendida, en parte porque coincide en algunos de sus usos con otra forma, dentro de lo que cabe, que posee mayor extensión. Sin embargo, resulta interesante mostrar el proceso de cambio semántico y los diversos contextos funcionales en que aparece, sobre todo porque su construcción: en lo que +verbo, se utiliza como patrón para generar otros complementos de referencia que terminan desarrollando un contenido procedimental.

\section{CONCLUSIÓN}

Las características de esta construcción preposición en+ estructura de relativo sustantivada (lo que cabe) son las de una estructura fijada, tiene valor como grupo, constituyen una unidad fraseológica que desarrolla un contenido procedimental: señala una limitación del ámbito de aplicación, una limitación de la aserción y una rebaja de la fuerza argumentativa de una valoración.

Puede aparecer antepuesta o pospuesta a su alcance. Aparece ya como grupo entonativo aislado, entre pausas, aunque también hay casos en que no se marca este aislamiento. Encontramos ejemplos pospuestos o intercalados en enunciados parentéticos. Incluso tenemos un caso en que aparece solo, sin la valoración, que se presupone en la respuesta, dada la alta frecuencia de uso con el adjetivo bien: bien en lo que cabe- pues en lo que cabe, con elipsis.

Diríamos, pues, que es un operador fijado ya con este contenido limitador que surge de su función como complemento de ámbito, función con la que coexiste en la actualidad. En otros contextos mantiene el valor de posibilidad de su base léxica (caber).

\section{REFERENCIAS}

ANSCOMBRE, Jean Claude y DuCROT, Oswald (1983): L'argumentation dans la langue, Liège, Pierre Mardaga.

AvanzI, Mattieu (2017): "Regards croisés sur la notion de macro-syntaxe", Travaux neuchâtelois de linguistique 47, 39-58.

Berrendonner, Alain (1990):"Pour une macrosyntaxe", Travaux de Linguistique 21: 25-36.
BerREndonner, Alain (2002): "Morpho-syntaxe, pragma-syntaxe, et ambivalences sémantiques", en H.Leth Andersen y H.Nolke (eds.), Colloque international, Macro-syntaxe et macro-semantique, Berna, Peter Lang, 23-41.

BERRENDONNER, Alain (2003): "Eléments pour une macro-syntaxe. Actions communicative, types de clauses, structures périodiques", en A.Scarano (ed.), Macro-syntaxe et 
pragmatique. L'analyse linguistique de l'oral, Roma, Bulzoni, 93-109.

BLANCHE BENVENISTE, Claire (2002): "Macro-syntaxe et micro-syntaxe: les dispositifs de la rection verbale", en H. Leth Andersen y H. Nolke (eds.), Colloque international, Macro-syntaxe et macro-semantique, Berna, Peter Lang, 95118.

Blanche Benveniste, Claire (2003): "Le recouvrement de la syntaxe et de la macrosyntaxe" en A. Scarano (ed.), Macro-syntaxe et pragmatique. L'analyse linguistique de l'oral, Roma, Bulzoni, 53-75.

BRINTON, Laurel (2010): "Discourse Markers", en A. Jucker e I. Taatvisainen,

Historical Pragmatics. Berlin, De Gruyter Mouton, 285-314.

BRIZ, Antonio, Pons, Salvador; PORTOLÉs, José (2008-actualidad): Diccionario de partículas discursivas del español, www.dpde.com

ClARIDGE, Claudia and Leslie ArNovick (2010): "Pragmaticalisation and Discursisation", in A.H. Jucker and I. Taavitsainen (eds), Historical Pragmatics, Berlin, De Gruyter, 165-92.

COMPANY, Concepción (2004): "Gramaticalización por subjetivización como prescindibilidad de la sintaxis", Nueva Revista Filología Hispánica 52 (1), 1-27.

DEHE, Nicole (2009): "Clausal parentheticals, intonational phrasing, and prosodic theory", Journal of Linguistics, 45, 569-615.

DEULOFEU, José (2016): “La macrosyntaxe comme moyen de tracer la limite entre organisation grammaticale et organisation du discours", Modèles linguistiques: 135-166. URL: http://ml.revues.org/2040; DOI: 10.4000/ml.2040

DieWALD, Gabriele (2011): Pragmaticalization (Defined) as Grammaticalization of Discourse Functions.Linguistics 49(2), 365-390.

DIK, Simon C., 1997(1957). The theory of functional grammar.2nd ed. Berlin: Mouton de Gruyter.

DostiE, Gaetane (2004): Pragmaticalisation et marqueurs discursifs.Analyse sémantique et traitement lexicographique, Bruxelles, De Boeck-Duculot.

Ducrot, Oswald (1984): El decir y lo dicho, Buenos Aires, Hachette.

FAuCONNIER, Gilles (1975): "Polarity and the scale principle". In: Grossman, Robin E., San, L. James, Vance, Timothy J. (Eds.), Papers from the Eleventh Regional Meeting, Chicago Linguistic Society, April 18--20, 1975. Chicago Linguistic Society, Chicago, pp. 188--199.
Fuentes ROdRíGuez, Catalina (2003): "Operador/conector, un criterio para la sintaxis discursiva". Rilce 19 (1): 61-85.

Fuentes RodríGueZ, Catalina (2004): "Enunciación, aserción y modalidad: tres clásicos”. Anuario de Estudios Filológicos 27: 121:145.

FuENTES RODRíGuEZ Fuentes Rodríguez, Catalina (2012): "El margen derecho del enunciado". RSEL 42/2: 63-93.

Fuentes RodríGuez, Catalina (2014): "Comment clauses and the emergence of new discourse markers: Spanish lo que es mas", Journal of Pragmatics, 61 (1), 103-119.

Fuentes RODRíGueZ, Catalina (2016): “Los marcadores de límite escalar: argumentación y "vaguedad" enunciativa", RILCE, 32, 1, 106133

Fuentes RodríGuez, Catalina (2017a(2000)): Lingüística pragmática y análisis del discurso. Madrid: Arco Libros, $3^{\mathrm{a}}$ ed.

FUENTES RODRÍGueZ, Catalina (2017b): "Macrosintaxis y lingüística pragmática". Círculo de Lingüística Aplicada a la Comunicación (CLAC) 71, 5-34.

Fuentes RodríGuez, Catalina (2018a): Diccionario de conectores y operadores del español, Madrid, Arco Libros, $2^{\mathrm{a} e d .}$

FUENTES RODRÍGUEZ, Catalina (2018b): Parentéticos, Madrid, Arco Libros.

Fuentes RodríGueZ, Catalina (2019a,e.p.): "Marcos asertivos- limitadores argumentativos"

Fuentes RodríGuez, Catalina (2019b, e.p.): "Atenuación y posibilidad".

Greenbaum, Sidney (1969): Studies in English adverbial usage, Londres: Longman.

GUTIÉRREZ ORdóÑEZ, Salvador (1997): "La determinación de los niveles oracionales", en S. Gutiérrez (ed.), La oración y sus funciones, Madrid, Arco/Libros, 368-426.

Hengeveld, Kees y MACKENZIE, J. Lachlan (2008). Functional discourse grammar. A typologicallybased theory of language structure. Oxford University Press.

KaltenBÖCK, Gunther, HeIne, Bernd y Kuteva, Tania (2011): "On Thetical Grammar", Studies in Language 35/4, 852-897.

LÓPEZ MARTín, José Manuel (2017): "Estudio macrosintáctico de las expresiones que introducen la fuente enunciativa: como.../ según.../ para.../ en palabras de.../ en opinión de...", Círculo de Lingüística Aplicada a la Comunicación (CLAC) 71, 99-114.

QuiRK, Randolph; GreEnbaum, Sidney; LEECH, Geoffrey y SVARTVIK, Jan (1972): A Comprehensive Grammar of the English Language, London: Longman, $2^{\mathrm{a}}$ ed. 
En lo que cabe y la limitación de la aserción | C. Fuentes

Real Academia Española (2017): Diccionario de la lengua española,

http://dle.rae.es/?id=DglqVCc . Último acceso: 17/3/2019.

TrAUGOTT, Elizabeth C. (1989): "On the Rise of Epistemic Meanings in English: an Example of Subjectification in Semantic Change", Language 65, 31-55.
TraugotT, Elizabeth C. (1995): The Role of the Development of Discourse Markers in a Theory of Grammaticalization.

http://web.stanford.edu/ traugott/ectpapersonline.html >. Última consulta: 05-052015. 